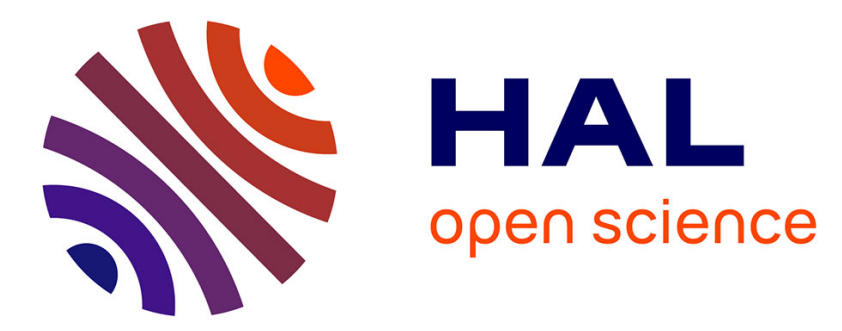

\title{
L'ÉTAT, L'EXPERT ET LE NÉGOCIANT : LE RÉSEAU DE LA " SCIENCE DU COMMERCE" SOUS LOUIS XV
}

\author{
Arnault Skornicki
}

\section{- To cite this version:}

Arnault Skornicki. L'ÉTAT, L'EXPERT ET LE NÉGOCIANT : LE RÉSEAU DE LA “ SCIENCE DU COMMERCE " SOUS LOUIS XV. Genèses. Sciences sociales et histoire, 2006, 65 (4), pp.4-26. 10.3917/gen.065.0004. halshs-03220696

\section{HAL Id: halshs-03220696 \\ https://shs.hal.science/halshs-03220696}

Submitted on 22 Dec 2021

HAL is a multi-disciplinary open access archive for the deposit and dissemination of scientific research documents, whether they are published or not. The documents may come from teaching and research institutions in France or abroad, or from public or private research centers.
L'archive ouverte pluridisciplinaire HAL, est destinée au dépôt et à la diffusion de documents scientifiques de niveau recherche, publiés ou non, émanant des établissements d'enseignement et de recherche français ou étrangers, des laboratoires publics ou privés. 


\section{L'ÉTAT, L'EXPERT ET LE NÉGOCIANT : LE RÉSEAU DE LA « SCIENCE DU COMMERCE »SOUS LOUIS XV}

\section{Arnault Skornicki}

Belin | « Genèses »

$2006 / 4 n^{0} 65$ | pages 4 à 26

ISSN 1155-3219

Article disponible en ligne à l'adresse :

https://www.cairn.info/revue-geneses-2006-4-page-4.htm

Distribution électronique Cairn.info pour Belin.

(C) Belin. Tous droits réservés pour tous pays.

La reproduction ou représentation de cet article, notamment par photocopie, n'est autorisée que dans les limites des conditions générales d'utilisation du site ou, le cas échéant, des conditions générales de la licence souscrite par votre établissement. Toute autre reproduction ou représentation, en tout ou partie, sous quelque forme et de quelque manière que ce soit, est interdite sauf accord préalable et écrit de l'éditeur, en dehors des cas prévus par la législation en vigueur en France. Il est précisé que son stockage dans une base de données est également interdit. 


\section{ฯ L'État, l'expert et le négociant: le réseau de la «science du commerce» sous Louis XV}

\section{Arnault Skornicki}

PP. 4-26

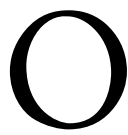
n ne parlait guère d'«expert» ou d'«expertise» sous l'Ancien Régime pour désigner un spécialiste d'un domaine chargé (par l'État ou une autre institution) d'étudier et d'évaluer les faits qui lui étaient soumis, dont les compétences scientifiques et techniques étaient requises en raison de la division toujours plus poussée du travail social, politique ou administratif. L'adjectif «expert» concernait d'abord les activités pratiques ou «mécaniques» qui avaient rapport au corps, et non les activités de l'esprit ou «libérales». Il qualifiait donc un homme «fort versé, fort expérimenté en quelque art qui s'apprend par expérience. Il est fort expert en Chirurgie» (Dictionnaire de l'Académie française 1762 : 699). Le substantif, lui, renvoie à partir du XVIIIe siècle à la seule expertise judiciaire, "Des gens nommés par autorité de Justice, ou choisis par les Parties intéressées, pour examiner, pour estimer certaines choses, \& en faire leur rapport» (ibid.; Rey 1992, 1: 762). Quant au conseil économique, militaire ou scientifique, il s'agissait moins d'un exercice de science appliquée que d'«administration» ou de "police» (compris comme la garantie du bien public dans tous ses aspects), d'autant plus que les procédures de recrutement de ces serviteurs de l'État n'étaient guère "méritocratiques» au regard de nos critères actuels (reconnaissance de diplômes officiels, réussite au concours). La détention d'un office vénal héréditairement transmissible, doublé du capital culturel légitime qu'étaient la compétence juridique et la culture classique, qualifiaient la plupart des prétendants au service de l'administration royale, à savoir les noblesses de robe et de finance et les autres officiers, à côté des commissaires et commis, plus maniables. Ainsi, en matière économique, devenue centrale avec les besoins financiers croissants de la Couronne, le recrutement des officiers se faisait largement à l'intérieur dudit corps et fort peu chez les professionnels du monde économique (banquiers, négociants, manufacturiers, fermiers) ${ }^{1}$. Et s'il existait bien des théoriciens en éco- 
nomie politique, ils se recrutaient d'abord dans les élites juridico-administratives et dans la république des lettres (Théré 1984). Marchands et manufacturiers restaient largement à l'écart des leviers de commande politique, relégués dans la basse culture du negotium loin du l'otium (Roche 1988 : chap. XII).

Pour des prétendants moins légitimes aux grandes charges de l'État, il convenait dès lors de mettre en avant des compétences qui faisaient défaut aux concurrents et passer par des circuits périphériques au pouvoir central, à savoir l'espace public littéraire. Telle fut la stratégie d'avancement social de plusieurs jeunes provinciaux montés à Paris dans les années 1750 et qui se réunirent autour de Jacques Claude Marie Vincent de Gournay. Tel est ce qu'on a coutume d'appeler aujourd'hui, un peu hâtivement, le "groupe de Gournay», du nom de cet intendant $d u$ commerce tenu pour un père fondateur $\mathrm{du}$ «libéralisme» et inventeur de ce qui en deviendra le slogan: «laisser faire, laisser passer». Longtemps occulté ou minoré dans l'histoire de la pensée économique, et a fortiori politique, maintenant largement connu depuis l'article pionnier d'Antoin Murphy (1986) et les travaux approfondis de Simone Meyssonnier (1989), de Catherine Larrère (1992 : chap. IV) et, plus encore, de Loïc Charles (1999 : partie II ; 2004; 2006), ce réseau mit à l'ordre du jour politique et mondain l'économie politique en France, et cela avant les physiocrates. Bourgeois d'Ancien Régime, nullement révolutionnaires quoique audacieux «améliorateurs», ils furent porteurs d'un projet, non de désintégration de la monarchie, mais d'intégration des groupes sociaux productifs dans la société de cour, fut-ce en redessinant les frontières qui séparent les ordres.

Dans quelle mesure leur «commerce politique» qui, en réponse à une demande politique, produisit une montée en généralité, comme on monte à Paris, pour opérer une conversion du regard sur l'ensemble du royaume, peut-il être envisagé comme un épisode particulier dans la formation de l'expertise économique, dans une dynamique de construction de l'État moderne, centralisé et administratif par lequel le roi magicien devient un roi technicien (selon l'expression d'Alain Guery)? Nous esquisserons la genèse sociale de cette œuvre collective que fut la science du commerce, en se demandant comment elle remodela la figure de l'administrateur par le réinvestissement de savoirs et savoir-faire négociants.

\section{Genèse et structure d'un réseau}

Le groupe de Gournay passe aujourd'hui pour une évidence historiographique. Les listes de noms, quelque peu variables, établies par les historiens de la pensée économique agrègent une quinzaine de personnalités diverses, pas toujours clairement liées à Gournay, et qui rassemblent tout autant ses supérieurs hiérarchiques que ses obligés, des prétendants à l'administration comme aussi des hommes de lettres. Les contemporains, par contre, soit évoquaient en termes 
vagues cette mobilisation intellectuelle ${ }^{2}$, soit ne donnaient pas toujours les mêmes noms, évoquant parfois les ramifications internationales du réseau ${ }^{3}$. L'incertitude sur les frontières de ce groupe et l'hétérogénéité des rapports sociaux qui semblent le tisser peut légitimement faire douter de son unité et de sa densité. Nous ne parlerons donc pas d'un "groupe» de producteurs intellectuels clairement délimité, à l'identité affichée, avec sa clôture sociale, ses droits d'entrée, ses lieux de rencontre, son organe de presse, ses stratégies de labelisation, comme ce fut le cas des physiocrates, «secte» fortement structurée. Ce qui réunit le groupe dit de Gournay est d'abord une marque politico-littéraire avancée dans une série de publications dès la décennie 1750 , la «science du commerce» ou «commerce politique», et un certain nombre de motifs rhétoriques et intellectuels qui définissent un voisinage social et intellectuel. Nous parlerons donc certes du «cercle de Gournay» 4 qui aurait pour centre l'intendant du commerce, mais aussi d'un réseau plus large où l'on peut distinguer cette clique d'interconnaissances mutuelles patronnée par l'intendant; des hommes de lettres qui rallièrent opportunément la cause du commerce politique sans être toujours personnellement liés à Gournay; et des ramifications multiples et imprévues dans lesquelles certains acteurs, parfois institutionnels, se réapproprièrent la science du commerce sans avoir jamais connu Gournay, dans un réseau d'intertextualité constituant une communauté de références intellectuelles au-delà des simples interactions directes entre individus (Van Damme 1998). Par «réseau Gournay», nous entendrons donc certes le réseau de Gournay (son capital social spécifique ou réseau «egocentré») 5 , mais aussi les diverses chaînes d'interdépendance au croisement desquelles on rencontre l'intendant du commerce, à titre d'intermédiaire, et dont le déroulement aboutit à la production d'une ouvre éminemment collective.

Le tableau ci-après qui s'inspire librement des principes que Howard Becker (1988) mit en œuvre pour analyser «les mondes de l'art» donne un aperçu partiel de la composition et de l'organisation du réseau.

\begin{tabular}{|c|c|c|c|}
\hline $\begin{array}{l}\text { Auteur } \\
\text { cardinal }\end{array}$ & $\begin{array}{l}\text { Patronage } \\
\text { politique }\end{array}$ & $\begin{array}{l}\text { Personnel de renfort } 6 \\
\text { administratif }\end{array}$ & $\begin{array}{l}\text { Personnel de renfort } \\
\text { littéraire (abbés) }\end{array}$ \\
\hline $\begin{array}{l}\text { Vincent } \\
\text { de Gournay }\end{array}$ & $\begin{array}{l}\text { Trudaine de Montigny } \\
\text { (intendant des Finances) } \\
\text { Malesherbes } \\
\text { (directeur de la Librairie) }\end{array}$ & $\begin{array}{l}\text { Véron de Forbonnais (14) } \\
\text { Butel-Dumont (9) } \\
\text { Clicquot-Blervache (4) } \\
\text { Abeille (6) } \\
\text { Plumard de Dangeul (2) } \\
\text { Turgot (4) } 7 \\
\text { Montaudouin de la Touche (3) } \\
\text { O'Heguerty (2) } \\
\text { [Herbert (1)] }\end{array}$ & \begin{tabular}{|l} 
Morellet (5) \\
Coyer (3) \\
Le Blanc (3) \\
Gua de Malvès (2)
\end{tabular} \\
\hline
\end{tabular}


Cette agrégation de noms est pondérée par l'importance de chacun (dans l'ordre décroissant) mesurée en fonction, d'une part de l'intensité des liens personnels avec Gournay, d'autre part du nombre (entre parenthèses) et de l'importance de leurs publications (en fonction des rééditions et recensions dans la presse $\left.^{8}\right)$ relevant de la «science du commerce» dans les décennies 1750-1760.

\section{Vincent de Gournay et ses jeunes prétendants à l'administration}

Le profil social du marquis Vincent de Gournay donne une bonne idée des trajectoires de plusieurs de ses jeunes collaborateurs9.

\section{Jacques Claude Marie Vincent, marquis de Gournay (1712-1759)}

Né à Saint-Malo, l'un des tout premiers ports de commerce français, d'un père armateur, appartenant à la petite noblesse terrienne par sa mère, Jacques est d'abord un héritier de cette grande bourgeoisie du capitalisme marchand, familial et patrimonial, distinct du capitalisme industriel concentré qui triomphera au xIxe siècle. II reçoit une véritable éducation aristocratique au prestigieux collège de jésuites de La Flèche, en vertu d'une stratégie familiale d'intégration de la société de cour au prestige toujours intact (Lespagnol 1995). Cependant, après sa scolarité, il entre dans les affaires familiales et part à dix-sept ans faire son apprentissage à Cadix: il y resta quinze ans et connut une belle ascension dans le milieu professionnel local (Turgot 1997 : 123-124). Jacques rentre au pays en 1744 pour discuter à Paris de "quelques entreprises de commerce» (ibid: 126) avec le gouvernement, probablement à l'occasion de "l'affaire des retours», c'est-à-dire d'un plan de rapatriement de fonds français d'Amérique espagnole en France (Meyssonnier 1989 : 169-173). C'est alors que le ministre de la Marine, Jean Frédéric Phélypeaux de Maurepas, lui confie une mission de renseignements sur les ennemis et alliés de la France en pleine guerre de succession d'Autriche (1740-1748), en Angleterre, Hollande, Allemagne (Schelle 1984 : 22-24): Gournay emploie sa qualité d'homme d'affaires comme une couverture afin d'observer, comparer et analyser les pratiques économiques différentielles des puissances voisines pour le compte du gouvernement, notamment pour découvrir le "secret» de la réussite britannique. Ayant bien mérité de la patrie comme "espion» économique, et après avoir hérité de son associé Julien Floran Jamets de Villebarre le marquisat de Gournay et épousé sa veuve, il se vit promettre une charge d'intendant du commerce après le retour de la paix en 1748. II se fixa à Paris, prit le titre de marquis, et paracheva la conversion de ses capitaux économiques en capital symbolique et politique par l'achat d'une charge de conseiller au Grand Conseil en 1749. Devenu officiellement intendant du commerce en 1751, il partagea son temps entre Paris, son domaine de Gournay et ses tournées en province. II démissionna en 1758 en raison de problèmes de santé et, sans doute aussi, par découragement.

Fort de son double capital culturel de négociant provincial cosmopolite et de gentilhomme formé aux humanités classiques, le nouveau haut fonctionnaire de l'administration économique centrale, porteur d'un audacieux projet réformateur ${ }^{10}$, réussit pleinement son insertion dans la société de cour louisquinzienne. Il demeurait cependant isolé au sein de l'intendance du commerce où ses trois collègues (François Boula de Quincy, Nicolas Doublet de Persan et Jacques Maris Jérôme Michau de Montaran), tous magistrats issus de lignées d'officiers, étaient partisans d'une «économie de police» plus traditionnelle et disposaient d'une faible culture économique ${ }^{11}$. Il bénéficiait en revanche de l'appui de son supérieur direct, Jean Charles Philibert Trudaine de Montigny, et 
de son équipe d'ingénieurs et savants dévolus à sa politique industrielle, cette dernière étant connexe à la clique Gournay en vertu d'une division du travail interne au Bureau du commerce entre les experts dans le textile et la chimie d'une part ${ }^{12}$, et, d'autre part, les conseillers économiques. Protégé, donc, par le puissant intendant des Finances, directeur des Ponts et Chaussées et membre éminent de l'Académie des sciences, ainsi que par le directeur de la Librairie Malesherbes qui favorisa la publication des œuvres du collectif - l'intendant du commerce de par sa position sociale avantageuse fut au principe de la dynamique de rassemblement de sa clique. C'est pourquoi de jeunes talents recrutés pour la plupart dans le milieu négociant de l'Ouest (Bretagne et Sarthe, régions manufacturières ouvertes sur la mer), tous scolarisés au collège, se rassemblèrent autour de lui. En dehors du cas particulier d'Anne Robert Jacques Turgot, et à part George Marie Butel-Dumont (Parisien) et Louis Paul Abeille (fils d'ingénieur du roi), tous viennent de province et du grand commerce maritime. La plupart sont roturiers, Gournay et Louis Joseph Plumard de Dangeul n'ayant guère que des quartiers de petite noblesse urbaine.

La clique Gournay (entendue comme réseau d'interconnaissances réciproques subordonnées à l'intendant du commerce) comprend huit acteurs. La propriété sociale distinctive de cinq d'entre eux est leur provenance sociale de négociant et/ou de fils de négociant (qui pratiquent le commerce en gros), fait exceptionnel pour les auteurs économiques du XVIII siècle: Gournay bien sûr, mais aussi Simon Clicquot-Blervache (1723-1796)13, d'une grande famille marchande de Reims, qui renonce aux affaires familiales pour s'installer à Paris et publie trois brochures dans la décennie 1750, dont au moins une en étroite collaboration avec Gournay, qui devint la grande référence de la critique libérale du monde corporatif ${ }^{14}$. François Véron Duverger de Forbonnais (1722-1800)15, dont la trajectoire est la plus homologue à celle de Gournay, reste le plus productif et le plus célèbre parmi les négociants de la clique: issu d'une grande famille de manufacturiers du Mans, il reçut une éducation aristocratique avant de travailler quinze ans dans le commerce d'exportation, pour lequel il séjourna en Italie et en Espagne. Renonçant aux affaires en 1753, il s'installe à Paris et se lia avec Gournay ${ }^{16}$. Devenu collaborateur de l'Encyclopédie pour les questions de commerce, il publie durant toute la décennie de nombreux textes, dont une traduction d'un classique de la pensée économique espagnole (la Théorie et pratique du commerce et de la marine de Geronymo de Ustáriz, 1753)17, dans la préface duquel il annonce la parution imminente d'une autre traduction, celle du Rétablissement des manufactures et du commerce d'Espagne de Bernardo de Ulloa (1753), par son cousin Plumard de Dangeul. On sait bien moins de choses sur ce dernier: issu du même milieu de négociants manceaux, il s'installe à Paris vers 1750 et acquiert une charge de parlementaire à la Chambre des comptes ${ }^{18}$ avant, semble-t-il, de jouer comme Gournay l'espion en arpentant l'Europe de 1754 à 175819. Et c'est bien Gournay qui «[...] encouragea Dangeul à publier Les 
Avantages et désavantages de la France et de l'Angleterre [...] et fit publier à Forbonnais les Éléments du Commerce» (Morellet 2000 : 72). Les deux cousins offrent un exemple remarquable d'hybridation culturelle, entre otium et negotium, où la culture classique se mêle à une culture économique tout à la fois pratique, théorique et «internationale». Les inventaires de leurs bibliothèques révèlent des goûts assez homologues 20 : celle de Forbonnais, par exemple, comporte les grands classiques de l'honnête homme du XVIII siècle (Jean Bodin, Jacques Bénigne Bossuet, Henri de Boulainvilliers, Fénelon, Montesquieu, abbé de Saint-Pierre...), mais aussi des manuels de commerce et des ouvrages d'économie théorique (Jacques Savary, Vauban, Richard Cantillon, Charles Davenant...), ainsi que de nombreux ouvrages étrangers (vingt-quatre britanniques et quarante-huit italiens dont la plupart en langue originale, plusieurs ouvrages espagnols). Quant à Jean Gabriel Montaudouin de la Touche (1722-1781), négociant gentilhomme nantais, riche armateur, il publia peu, mais commit un éloge funèbre de Gournay (Journal de commerce 1761 : 71-85) et participa avec lui à la création de la première Société d'agriculture.

Trois juristes, ensuite, furent tout aussi proches de l'intendant du commerce. Abeille fut ainsi son secrétaire personnel, publia dans les années 1760 sur le commerce du blé et fit une brillante carrière au Bureau ${ }^{21}$. Butel-Dumont, après une expérience de diplomate à Saint-Pétersbourg, devint chargé de dépôt du Contrôle général. Il aide Gournay à traduire les Traités sur le commerce de Josias Child (1754)22, et de son propre aveu, c'est sur le conseil avisé d'«hommes éminents» qu'il publia en 1754 une traduction de John Cary et une Histoire et commerce des colonies angloises, qui lui ouvrirent les portes de l'administration 23 . Enfin, the last but not the least, Turgot, jeune seigneur promis à un grand avenir, qui se dira disciple de Gournay autant que de François Quesnay, fut au début de sa carrière un proche collaborateur du premier: il l'accompagna dans ses tournées d'inspection en province pendant trois ans (de 1753 à 1756). Le jeune maître des requêtes traduit ainsi un texte de Joshua Tucker (1755), que Pierre Samuel Dupont de Nemours (1844) comptait dans le réseau Gournay. Que Turgot rédigeât l'éloge funèbre de son maître en 1759 , recueillant les données biographiques auprès de ses proches, indique la proximité entre les deux hommes.

En revanche, deux personnages ne semblent pas clairement liés à Gournay et sa clique, sinon par des rapports de voisinage textuels bien attestés par la presse de l'époque qui les associe fréquemment ${ }^{24}$ : Pierre André O'Heguerty (17001763), procureur général au conseil supérieur de l'île Bourbon, pratiqua le commerce avec les Antilles et, une fois revenu en métropole, publia en 1754 et 1757 deux ouvrages sur la «science du commerce maritime» (O’Heguerty $1754: 3$ ) qui illustrent les préoccupations et positions du groupe au-delà des savoirs pratiques de l'armateur (mécanique, géométrie, astronomie) et renvoient à Forbonnais et Claude Jacques Herbert. Enfin, le succès de l'Essai sur la police générale des 
grains (1753) du dernier, personnage mal connu, fut également associé à la vogue de la science du commerce par la presse de l'époque.

Hormis Montaudouin de la Touche qui persévéra dans le grand négoce nantais, et O'Heguerty qui avait déjà une carrière bien remplie, tous se reconvertirent dans l'administration au Bureau du commerce (à l'inspection des Manufactures, ouverte aux roturiers 25 , ou à l'inspection des Monnaies pour Forbonnais, qui devint le principal conseiller du contrôleur général Étienne de Silhouette en 1759) ou dans une juridiction traitant des affaires économiques (Plumard de Dangeul à la Chambre des comptes, avant de devenir maître d'Hôtel de la maison du roi-Journal ceconomique $1755: 21$ ), après avoir publié sur le «commerce politique», attestant de leurs compétences économiques distinctives ${ }^{26}$.

\section{Le personnel de renfort littéraire et la «mondanisation» de l'économie politique}

$\mathrm{Au}$-delà des collaborateurs de Gournay, un personnel de renfort plus spécifiquement littéraire s'associa à cette entreprise sans toujours entretenir avec lui de liens intenses. Ce qu'ils partagent avec le personnel de renfort administratif, c'est un certain fonds culturel, un même «inconscient d'école» hérité du collège, qui leur permit d'ajuster leur conversation aux normes curiales et de s'insérer dans les salons. Ce capital culturel spécifique n'était cependant pas investi dans le même espace social, ni avec la même stratégie et des dispositions identiques. Les abbés, sans fortune ni emploi fixe, ambitionnaient une place dans une académie ou une pension royale régulière afin d'échapper à la précarité économique et symbolique inhérente à la condition de la majeure partie des hommes de lettres des Lumières, tandis que les riches négociants et magistrats tentaient d'intégrer les élites nobiliaires de l'État royal français. Les premiers veulent atteindre les sommets de la république des lettres (André Morellet à l'Académie française y parviendra), quand les seconds n'y transitent que pour s'ouvrir le chemin conduisant à la haute administration27. Si appartenir à l'Académie des sciences comme Jean Paul Gua de Malvès (1757), d'extraction modeste, le préservait quelque peu de la précarité, ses confrères avaient plus intérêt encore à ajuster la science du commerce aux normes littéraires et à l'horizon d'attente imputés à la haute société aristocratique et curiale. Le ralliement de ces gens de lettres ressemble fort à une transaction d'intérêts bien compris: également provinciaux et roturiers, mais issus de familles bien moins favorisées, ils empruntèrent la voie cléricale pour financer leurs études; hommes de lettres avant la constitution d'un champ littéraire autonome, ils restaient largement dépendants des Grands et de la Couronne pour consolider leur situation. La science du commerce, loin de se réduire à un discours d'expertise d'État, répondait ainsi à une nouvelle demande mondaine (dans les salons et un marché éditorial restreint mais en expansion) en matière de «sciences, arts et métiers» que le mouvement encyclopédique avait intégrée et intensifiée. La contradiction entre le sérieux et le mondain fut résolue par nos abbés dans un traitement léger et frivole des matières utiles, sans pédan- 
terie ni technicité, proscrites à la cour comme dans les salons. C'est pourquoi l'économie politique se déclinait aussi volontiers sous les genres de la brochure polémique, voire du conte satirique comme le Chinki (1768) de Gabriel François Coyer, littérateur plaisant autant que moraliste intransigeant. Mais ce dernier devint un promoteur de la science du commerce en publiant sur le conseil de Gournay ${ }^{28}$ ce qui devait rester le plus célèbre de ses ouvrages, La noblesse commerçante (1756), qui cristallisa à merveille les questions politiques soulevées par le réseau et provoqua une célèbre controverse: en proposant d'abolir la loi de dérogeance, Coyer redéfinissait autant la noblesse qu'il n'anoblissait le commerce, en honorant l'intérêt et les marchands au sein de la communauté nationale, groupe social traditionnellement déconsidéré, mais principale source de puissance politique et de patriotisme alors que s'engageait la guerre de Sept Ans. Point sur lequel l'abbé Jean Bernard Le Blanc, déjà connu comme dramaturge et fin connaisseur de l'Angleterre, était en parfait accord: ses traduction et préface des Political discourses de David Hume, grand succès du philosophe écossais, contribuèrent elles aussi à «mondaniser» les thèmes de la science du commerce. Hume apparaît comme la grande figure étrangère du réseau Gournay, entretenant une correspondance avec Le Blanc, Morellet et Turgot; il deviendra un ami intime de Trudaine de Montigny (fils) lors de son séjour à Paris (1763-1766), capitale philosophique et détour obligé pour tout étranger désireux d'élargir son capital littéraire à l'échelle européenne. Quant à l'abbé Morellet, une des principales sources sur le réseau et figure éminente des Lumières européennes, on peut le compter comme le huitième et seul homme de lettres de la clique Gournay29, aux côtés de Forbonnais ou de Abeille; il destinera toute sa carrière au service de la science du commerce à ses talents de polémiste, lesquels lui firent endosser le surnom d'«abbé Mords-les» (Darnton 1992 : chap. III).

Trois grands lieux de sociabilité furent marqués par la présence du réseau Gournay. Côté savant, l'académie des belles-lettres et des sciences d'Amiens fit l'objet d'un investissement particulier, avec la bienveillance de l'ancien intendant de Picardie et nouvel intendant des Finances Germain Louis Chauvelin. Des prix mis au concours par la Société, Claude Carlier et Nicolas Desmarets (deux membres de l'équipe Trudaine) remportent ceux de 1751 et 1752 , ClicquotBlervache ceux de 1755, 1756 et 1757. Mais le réseau ne se contenta pas d'investir les sociétés savantes déjà existantes: il fut à l'origine d'un nouveau type d'académie, la Société d'agriculture, de commerce et des arts dont Montaudouin de la Touche adressa le projet aux États de Bretagne, et qui fut adopté en 1756 avec l'appui de Gournay ${ }^{30}$, lequel fit nommer Abeille (1760) secrétaire général: les préoccupations du negotium devenaient ainsi la raison d'être d'un haut lieu d'élection de l'otium. Sa composition sociale rompait avec les distinctions d'ordres pour faire prévaloir la logique de la compétence sur la hiérarchie des rangs (France. États provinciaux de Bretagne. 1757 : 4-5). Côté mondain, il est probable que le salon de Trudaine, très couru à Paris ou sur ses terres de Monti- 
gny, accueillit Gournay et ses amis. Et c'est l'intendant des finances qui introduisit Morellet dans le célèbre salon de Mme Geoffrin.

\section{Science du commerce, expertise et montée en généralité}

\section{Cultures négociantes et commerce politique: \\ le travail d' "expert» de Gournay}

Dotés des ressources culturelles légitimes, et du même coup se sentant légitimes à la prise de parole politique, une fois à Paris, lieu du pouvoir central et de l'édification de l'État moderne en France, les (ex)négociants du réseau offrent un cas intéressant d'appréhension élargie de la politique à l'échelle du territoire, conçu et souhaité comme homogène, fluide et désenclavé (Dockès 1969), lieu d'exercice du pouvoir d'État, garant de l'intérêt général, et dont le roi n'est guère que l'incarnation. Cette universalisation ne se produit certes pas par un brusque passage du pratico-local au théorico-national: ils arrivèrent à Paris préalablement remplis d'ambitions politiques et savantes fertilisées par leur éducation aristocratique et leur connaissance, plus originale, des économistes britanniques et espagnols. Cependant, leur nouveau statut de prétendant à l'administration fut la condition pour que ce qu'ils portaient en puissance ait la possibilité de passer à l'acte (théorique). Nous partirons du travail d'administrateur de Gournay pour arriver à la manière dont celui-ci fut repris, formalisé et publiquement promu par son «réseau».

Avec la création du Bureau du commerce sous Louis XIV, les représentants de la France industrieuse et marchande firent leur entrée dans l'administration centrale avec les douze députés des places de commerce, à titre de pouvoir consultatif. Une telle audience inaugura une circulation à double sens, de Paris vers la périphérie et des représentations des marchands et manufacturiers vers le sommet de l'État royal (Smith 2003). L'accumulation des savoirs négociants sous l'Ancien Régime est un phénomène bien connu des historiens, notamment grâce aux manuels de pratique commerciale, à vocation pédagogique ou documentaire, qui reflètent assez fidèlement les formes de l'activité et de pensée marchandes (Jeannin 1989; Van Damme 2005 : 50-58). Avec Gournay, on a une contribution qualitativement distincte, qui s'efforce de poser «la science du commerce» en l'opposant au savoir du négociant, comme à l'économie de police traditionnelle. La science du commerce, en effet, «n'est donc autre chose que de savoir tirer parti des avantages de son pays, d'y mettre l'argent et les hommes en action, et les terres en valeur» (Tsuda $1983: 285$ )31. Elle n'est nullement celle des technologies de contraintes, dissuasion et punition à l'adresse des marchands de toute espèce dont on ne se défierait jamais assez, comme le veut la théorie de la police. Elle n'est pas non plus réductible au savoir-faire des commerçants, comme s'il suffisait de les «laisser faire», et de leur déléguer la politique économique sans qu'ils le sachent pour 
atteindre l'équilibre optimal. Mettre «l'argent et les hommes en action», c'est mener une politique incitative et non coercitive (par la baisse du taux d'intérêt, par l'anoblissement du négociant); et cela, seule la puissance publique dispose des moyens symboliques et macro-économiques pour les mettre en œuvre. Seule une science d'État peut se charger de rendre au commerce sa liberté et sa vigueur, de produire la liberté (Foucault 2004 : 69) en indiquant les moyens de lever activement les contraintes qui pèsent sur la production de richesses.

Mais comment s'opère cette conversion du regard dans le travail politicoadministratif de Gournay, cette reconversion d'une culture négociante dans la politique, dans la perspective d'une histoire concrète de l'abstraction (Perrot 1992 : 19)? Parmi les compétences négociantes (Jeannin 1995), il convient de distinguer: 1) la connaissance proprement empirique des marchandises, que seul procure un long apprentissage; 2) des connaissances plus scientifiques comme l'arithmétique, la géographie appliquée ou le droit commercial, à finalité utilitaire; 3) une culture sur le «commerce en général» relevant tant de l'éthos de négociant que d'une théorie économique plus formalisée, largement empruntée à des sources britanniques, hollandaises et espagnoles, alors peu connues en France. Cette dernière strate fait que Gournay n'était sans doute pas ignorant de toute politique avant d'y entrer. Et sa brève carrière d'«espion économique " constitua une politisation spécifique qui le sensibilisa durablement à la politique internationale ${ }^{32}$, en même temps que s'édifiaient de nouveaux savoirs patriotes du côté de la technologie industrielle (notamment textile). La combinaison des trois compétences lui permit de lier le particulier et le général tout autrement que ne le fit l'économie de police. Ainsi, dans ses nombreux rapports sur le commerce colonial, notamment du Levant et d'Amérique ${ }^{33}$, il recense avec un luxe de détails la diversité des marchandises et débouchés, et comme «expert» en commerce maritime il fait remarquer dans un épais mémoire que l'excès de matelots renchérit le coût du transport et pèse sur la marche du commerce français, laissant libre cours aux ambitions britanniques en Amérique ${ }^{34}$. Son expérience espagnole, par ailleurs, lui inspira deux mémoires, où il détaille l'économie du royaume catholique allié en quatre secteurs (fabriques, compagnies, banque et commerce des Indes), afin de préparer un traité de commerce pour contrer les ambitions anglaises à la fin de la guerre de succession d'Autriche ${ }^{35}$. De même, ayant observé depuis Cadix que les Silésiens se sont emparés du commerce des toiles à destination de la Guinée en imitant les toiles françaises tout en produisant à moindre coût, l'intendant demande à l'ambassadeur français à Hambourg des renseignements sur les techniques de fabrique en Silésie (Tsuda 1993 : 158). Relevons aussi que, fort d'un savoir-faire aussi important dans le métier de négociant que le maniement des changes, il recommande d'évaluer le taux de change franco-britannique «relativement à la valeur de l'argent dans ces deux Royaumes ", c'est-à-dire en fonction des prix comparés du métal dont sont faites les espèces de part et d'autre de la Manche ${ }^{36}$. 
La culture livresque internationale de Gournay constitua également un atout sérieux dans son travail d'intendant: le British Merchant de Charles King (1721) lui fournit ainsi des statistiques sur la consommation des toiles françaises en Angleterre (Tsuda 1993 : 60-61); et le Complete English Tradesman de Defoe (1715) lui donne une estimation de la proportion d'Anglais employés dans le commerce (ibid.: 50-51), obtenue grâce à l'arithmétique politique. Cette dernière, fondée par William Petty et Charles Davenant (lequel fut traduit par Forbonnais), étendait des méthodes éprouvées dans des secteurs d'activité précis (comme la médecine ou le négoce) pour calculer la population, et apparaissait comme une nouvelle forme d'expertise faite d'un langage articulé et de techniques précises à l'usage des gouvernants et soumise à l'examen du public, bien différente des grandes enquêtes françaises lancées secrètement depuis le pouvoir central (Desrosières 2000 : 34-37; Brian 1994 : 153-156). La fréquentation assidue des auteurs anglais et espagnols, ainsi que de Cantillon (dont Gournay fit publier l'Essai sur la nature du commerce en général en 1755), fournit à l'intendant les cadres théoriques de son expérience de négociant. L'éthos de ce dernier le disposait à exiger de l'État à la fois «liberté» et «protection», ce dont Gournay convenait volontiers ${ }^{37}$. Mais, bien loin de défendre cette liberté en termes individuels comme la plupart des négociants français qui ne se souciaient guère d'incohérence en défendant à la fois la liberté et l'exclusif colonial (Tarrade 1996), l'intendant, qui distingue l'intérêt du commerce de celui des commerçants, réclame l'abolition des privilèges en la matière et l'ouverture totale de la concurrence entre marchands français, mais français seulement. Autrement dit, il préconise un protectionnisme proprement national sur le modèle anglais du Navigation Act, en lieu et place du maintien d'un monopole d'une poignée de navigateurs privilégiés et d'actionnaires de la Compagnie des Indes. On a là un bon exemple de nationalisation de l'entendement politique, ou de passage de l'esprit de commerce à l'esprit d'État, qui élève à la dignité publique le «commerce en général» comme levier de la puissance politique sur la scène européenne, de sorte qu'il faut «faire prioritairement \& exclusivement à tout étranger le commerce de ses colonies \& y deffendre absolument l'introduction de toute marchandise étrangère ${ }^{38}$.

En revanche, pour ce qui est du marché intérieur, la libre concurrence doit devenir la règle. Tout le travail de l'intendant Gournay s'inscrit dans le projet de faire valoir le rôle social du négociant et l'utilité de la libre concurrence contre les monopoles détenus par les corps de marchands-fabricants ${ }^{39}$. Conseillers subalternes, les intendants du commerce furent cependant parmi les premiers animateurs du Bureau: traiter les requêtes et plaintes des corps de marchands, instruire les dossiers, préparer des rapports, suivre la correspondance et rédiger les arrêts soit tout le travail préparatoire législatif ${ }^{40}$. Ainsi, on observe constamment dans les rapports et correspondance de Gournay une montée en généralité à partir de cas d'espèce soumis à son expertise, proche de ce que Philippe Steiner (1998: 
chap. I), faisant sien le lexique wébérien, appelait la «rationalisation formelle» de la connaissance économique, caractérisée par le rejet explicite des valeurs. La correspondance et ses mémoires administratifs, qui témoignent de sa confrontation quotidienne aux requêtes des corps de métiers, sont truffés d'analyses de portée beaucoup plus générale. Comment, par exemple, juge-t-il les plaintes des sucriers d'Angers et de La Rochelle, qui au nom de leurs privilèges exclusifs, attaquent la compagnie de Jésus qui a fait construire une sucrerie (Tsuda 1993 : 64-79)? Un autre intendant aurait probablement mis en balance les arguments des deux parties avant de trancher de manière pondérée en fonction d'impératifs de justice et de police, dans l'intention de satisfaire les intérêts et du producteur et du consommateur afin de se rapprocher du juste prix, comme son collègue Michau de Montaran (1754) à propos du commerce des blés pour lequel il recommande une politique d'exportation limitée. Gournay, lui, se fait savant plutôt que juge, et subsume le cas d'espèce sous la problématique générale de la concurrence et des résistances qui lui sont opposées. S’il prend résolument le parti de la compagnie, c'est parce que, muni de la science du commerce, il démystifie la rhétorique dénonciatrice des marchands de défense de privilèges comme voile d'un intérêt égoïste mal compris et corporatif. Et si les compagnies se saisissent du Bureau, c'est bien parce que les moines, main-d'œuvre ascétique par éthos religieux, disciplinée et bon marché, les menacent par leur compétitivité imbattable (ibid.: 68-69). L'intendant évalue donc ce litige local précis non par souci d'établir une juste balance entre intérêts opposés pour satisfaire le bien public dont les magistrats revêtus de l'autorité souveraine sont garants, mais au nom d'un savoir performatif informé des lois de l'équilibre économique.

L'«expertise» ne se réduit pas ici à une compétence professionnelle reconnue par les pairs, mais à un transfert de compétences dans un autre monde social que celui du négoce, ajustées aux exigences de l'action publique. Il est vrai que dans les coulisses de la science du commerce, se profile un portrait du négociant moins flatteur que ne le suggère celui, publiquement mis en avant, de l'intrépide navigateur dévoué à sa patrie. Ainsi, le diplomate Pierre Michel Hennin se plaint de l'étroitesse d'esprit des marchands hollandais, rivés à leur quête de profit, incapables d'offrir une vue synoptique du commerce de leur nation ${ }^{41}$. Sollicité à titre d'« expert» économique, Forbonnais en convient volontiers et stigmatise leur «mauvaise foi» qui leur fait spolier méthodiquement le commerce des grandes nations territoriales: ce que la France pourrait aisément résoudre en restaurant une marine marchande et militaire offensive grâce à «un plan d'administration et des administrateurs actifs éclairés »42. Or, justement, la forteresse administrative occupée par les élites parisiennes ignorantes de la réalité du pays doit être décloisonnée, et il revient à la science du commerce de faire communiquer des mondes aussi éloignés que les négociants et les administrateurs. Alors que les finances publiques relevaient encore du secret d'État, Gournay appelle de ses vœux, la formation d'«un Bureau ou Conseil du commerce, où toutes les par- 
ties du commerce aboutissent, et où l'on voit tout objet du commerce» (Tsuda 1983 : 401). Pour décentraliser les catégories mentales des administrateurs, tout en concentrant les informations sur l'état du commerce dans chaque province, il convient de faire communiquer les bureaux pour mieux évaluer l'interdépendance des activités économiques nationales, afin de diagnostiquer l'état du royaume ${ }^{43}$. Pour ce faire, il faut rompre avec les pratiques et mentalités administratives qui mettent sous surveillance l'activité marchande, considérant que «[...] la tromperie étoit si fort inséparable du commerce que le souverain devoit veiller continuellement pour l'empêcher» (ibid.: 249). De sorte que la Couronne dépêche sur le terrain des "hommes sans connoissances des principes du commerce» (ibid.: 251-252) pour faire appliquer un règlement tatillon. Le parisianisme des dirigeants français, coupables de provincialisme intellectuel et de méconnaissance des pratiques politiques des voisins, fait que "peu de gens à Paris distinguent le négociant qui fait mouvoir les flottes, et qui en secourant les pays étrangers enrichit le sien d'avec le marché en détail», écrit-il à Maurepas (Tsuda 1993 : 10). Il convient donc d'élargir l'expertise économique au-delà du cercle des administrateurs, auprès de ceux qui ont une véritable expérience des affaires, afin que la bureaucratisation de l'État ne le coupe pas de ses forces vives.

\section{Une science patriote au miroir de l'Angleterre}

Sous l'impulsion parfois directe de Gournay ${ }^{44}$ qui, tenu à un devoir de réserve, publia peu et jamais sous son nom, les auteurs du réseau éditèrent plus de quarante ouvrages et traductions dans les décennies 1750 et 1760. La réussite fut au rendez-vous, et la mise au goût du jour politique et mondain de l'économie politique accomplie: "L'économie politique est aujourd'hui la science à la mode», lit-on dans le Mercure de France (1758 : 69) à propos d'un ouvrage de ButelDumont. Si l'espace public fut d'abord investi en raison de stratégies de promotion sociale, si la plupart des proches de Gournay cessèrent pratiquement de publier après avoir trouvé un emploi dans l'administration, la république des lettres n'en devint pas moins la caisse de résonance d'une nouvelle «expertise» qui décloisonna l'administration économique et leva le «secret du roi» en la matière. Il s'agissait à la fois de rendre publics les questions de finances royales et un nouveau mode d'expertise détaché des catégories de l'économie de police - suspicieuse envers le marchand, soucieuse de la qualité du produit et de l'intérêt du consommateur plutôt que de la quantité et de l'intérêt du producteur (Kaplan 1986 : chap. I et II). Nos prétendants à l'administration répondirent aux attentes de deux types de public: les responsables de la "police» économique du royaume, en droit d'exiger des preuves de leurs compétences; et le public lettré qui attendait qu'on mette à sa portée les débats politiques centraux du moment; ce à quoi répondait le programme de traduction de livres anglais qui épousaient l'anglomanie ambiante tout en exposant une critique de la monarchie dans des formes acceptables. Pour autant, cet entre-deux doit aussi se comprendre comme une cir- 
culation entre pratiques administratives et «littéraires». Gournay, ainsi, faisait circuler ses écrits en dehors du ministère, et en premier lieu ses Remarques sur Child, base de travail de ses collaborateurs ${ }^{45}$, notamment du livre de Clicquot-Blervache (1758) dirigé contre les compagnies et l'inspection des manufactures. ButelDumont, lui, fournit des sources chiffrées sur les finances royales depuis son poste d'archiviste au Contrôle général des finances (qui alimentèrent directement les Recherches et Considérations sur les Finances de la France de Forbonnais). En sens inverse, la mise sur le marché éditorial d'une nouvelle économie politique alimenta le travail des administrateurs comme Trudaine (Lebeau 2004).

La cohérence de la production intellectuelle du réseau tient à trois grandes thèses politico-économiques, toutes liées entre elles: la revalorisation du statut des commerçants, et surtout du riche négociant; la diminution du taux d'intérêt, lié à l'impératif de réorienter le capital vers les activités productives au détriment de la finance et des rentiers; la critique des monopoles et des corporations, assise sur une assez puissante analyse des bienfaits de la libre concurrence comme vecteur de l'allocation optimale des ressources - critique qui débouche sur un projet d'abolition des privilèges exclusifs accordés aux communautés de marchands. La science du commerce fut mise en scène par le réseau Gournay comme un savoir tourné vers l'action, éloigné de toute conception rigide de la science moderne (axiomatique, déductive, caractérisée par la certitude et formulant des lois invariables). Ainsi, Forbonnais (1980 : VII), opposé au dogmatisme des physiocrates, opte pour une démarche inductive et conjecturale, plutôt que déductive et réglée par une «évidence» pleine et entière, les principes abstraits ne jouant qu'un rôle heuristique dans l'observation des faits. Mais la science du commerce constitue cependant un saut qualitatif par rapport aux savoirs négociants, explique Morellet dans la présentation de son Dictionnaire de commerce, qui prend le contre-pied de ses prédécesseurs $(2000: 185)^{46}$. Au négociant, sont nécessaires la connaissance des faits particuliers (l'expérience au sens d'Aristote), et celle des opérations de commerce générales (la «science des comptes»). Mais à l'administrateur et l'homme de lettre, lesquels par leur capacité d'abstraction s'élèvent «au-dessus de l'usage \& de l'opinion [...]» (Morellet 1769 : 376), est nécessaire «la science de l'économie politique qui [...] s'efforce de déterminer les meilleures loix par lesquelles on peut diriger au plus grand bonheur des sociétés le Commerce» (ibid.: 325)47. Cette science n'était donc rien d'autre que celle du bien public, et ne cherchait en ce sens nullement à s'affranchir de la politique et de la morale. Rejetée dès lors dans l'ombre des savoirs profanes, la connaissance pratique des marchands prétendait pourtant elle aussi au titre de science, comme ce marchand bordelais qui rappelle à Malesherbes, peu avant l'entrée en scène de Gournay, que l'arithmétique marchande est bien «ce que l'on nomme la science du Commerce»48.

Il n'en demeure pas moins que Gournay projetait de donner la parole aux classes productives que la société aristocratique leur avait confisquée, et soumettre ainsi la politique économique à l'examen de «l'opinion publique» constituée en 
tribunal (d'expertise), non par goût démocratique pour la discussion, mais afin de rationaliser les procédures de décision en la soustrayant aux groupes sociaux qui ont intérêt au secret. Ainsi, la fameuse querelle des "toiles peintes»49 (toiles de coton imprimées dont l'importation était prohibée) eut un large écho public grâce à un ouvrage de Forbonnais (1755) auquel Gournay collabora. À leur suite, Morellet rendit publics les projets d'arrêt du Bureau et les avis des députés du Commerce pour former «une parfaite instruction» devant le «Tribunal du Public» (Morellet 1759 : 3). Cette conception de l'opinion publique dérive d'une certaine lecture de la vie politique britannique où les comptes de la nation sont discutés au grand jour au Parlement. Mais, en contexte monarchique français, l'opinion publique doit se nicher dans d'autres institutions «représentatives», peu "démocratiques» mais moins tumultueuses et plus raisonnables que la République insulaire (Bureau du commerce, cours souveraines, académies, presse...) ${ }^{50}$. Cette publicité des débats permettrait de canaliser l'expression des différents intérêts du royaume et de minimiser le risque d'erreur, car l'on se trompe moins à plusieurs qu'entre «bureaucrates» (néologisme inventé par Gournay) reclus dans leur forteresse parisienne (Plumard de Dangeul 1754 : 155).

Le réseau Gournay, par ses traductions et multiples analyses de la vie politico-économique britannique, fournit une contribution décisive aux transferts culturels qui autorisèrent la construction de la référence nationale anglaise. Définie comme «la mise en relation de deux systèmes autonomes et asymétriques» (Espagne et Werner 1988 : 5), la notion de «transfert culturel» a le double avantage de rendre à la circulation internationale des idées sa réalité à travers les divers supports matériels (voyageurs, livres, correspondances...), et de mettre en évidence les multiples transformations relatives au contexte de réception qui affectent nécessairement les éléments culturels. En revanche, elle a cet inconvénient de supposer des unités nationales relativement homogènes, alors même que, dans le cas qui nous occupe, il s'agit plutôt d'un circuit Londres-Édimbourg-Paris circonscrit aux élites lettrées avant la constitution des États-nations. L'année 1748, avec la publication de L'Esprit des Lois et le retour de la paix, inaugura une période d'intense anglomanie. Montesquieu (1979: XI, 6; XIX, 17) dessine le portrait d'une République moderne, libre, modérée et méritocratique, qui subordonne sa politique aux impératifs de son commerce. Préoccupés par la menace britannique 51 , Gournay et son réseau se positionnent relativement à Montesquieu (Charles 2006), sans s'aligner sur ses positions. Deux fois plus petite et trois fois moins peuplée, l'Angleterre n'en menaçait pas moins l'hégémonie de la France (Plumard de Dangeul 1754 : 47). Leur «anglomanie» admirative n'est pas anglophilique, mais stratégique: il s'agit de s'inspirer des pratiques de l'ennemi pour retourner ses armes contre lui (Tsuda 1993 : 37), ce à quoi était destinée la douzaine de traductions (fort libres) d'ouvrages anglais. Tel est le grand horizon géopolitique du travail d'administrateur de Gournay: même ses efforts les plus localisés pour promouvoir les bienfaits de la concurrence 
auprès des inspecteurs de Manufacture, intendants de province ou prévôts des marchands sont explicitement destinés à faire pièce aux Anglais ${ }^{52}$. La lecture des Britanniques constitua pour les auteurs du réseau Gournay, tous anglophones (par obligation professionnelle ou par anglomanie de lettrés), un utile prétexte pour exiger une réforme urgente du royaume, mettant en balance les réussites insolentes de la vigoureuse République et les «retards» de l'impotente monarchie. Le paradigme anglais permettait de mettre en contraste la dimension artificielle et superficielle de la «civilisation» à la française (brillante mais vaine), et le solide processus économico-technique de civilisation des Anglais (Le Blanc 1754, I: vi). L'utile est opposé au futile de la société de cour et des apparences, incapable de reconnaître la grandeur publique des activités mercantiles, leur rôle moteur dans une guerre devenue économique, la balance du commerce étant véritablement celle des pouvoirs (Tsuda 1983 : 301).

La «science du commerce» répondait à une demande publique et peut donc être tenue pour une forme d'expertise économique. Mais cette dernière n'apparaît ni comme une activité scientifique dégradée par son hétéronomie vis-à-vis des problématiques imposées par les dirigeants politiques, ni comme une science appliquée et technicienne. Elle participait au contraire au développement effectif de la science et de ses procédures d'universalisation, car la conformation à la demande étatique obligeait nos anciens négociants à élargir leur horizon épistémologique et à considérer le commerce «en général», comme disait Cantillon, à resituer les opérations particulières du commerce dans le système général de production et d'échanges. À l'exemple de la statistique, de l'arithmétique politique ou des sciences camérales, l'élévation épistémologique d'une science d'État comme l'économie politique inquiète la distinction préconstruite entre savoirs savants et savoirs experts, à une époque où il n'y avait nulle constitution d'un secteur autonome d'activités en théorie économique ${ }^{53}$. Et, à la différence de l'expertise industrielle qui renvoie à des compétences techniques spécialisées sur la nature ou les artefacts, «l'expertise» en économie publique relevait directement de la pensée et de la pratique politiques, nos experts s'apparentant davantage à des «conseillers", non certes du prince, mais comme des «administrateurs», figure encore trop minorée dans l'histoire de la science (économique) ou reléguée au second plan parmi la cohorte d'«experts de l'administration » aux vues étroites (Schumpeter 1983). Si science économique il y avait en effet, ce n'était guère comme discipline universitaire où n'existait alors aucune chaire de cette sorte (même si un corpus de références spécifique commençait à se constituer ${ }^{54}$, mais comme science d'État ici retrempée dans les savoirs négociants. À la figure judiciaire de l'officier habilité à exercer la justice du roi dans la confidentialité de la raison d'État, se substitue l'administrateur savant qui forme ses 
décisions non comme des jugements axiologiques mais des jugements de connaissance fondés dans une science informée par une discussion publique ouverte - soit une forme d'expertise civique collective.

Un an après la mort de Gournay, la frange «libérale» de l'administration centrale était en passe de dominer le Bureau du commerce; le contrôleur général Bertin étendit le principe des Sociétés d'agriculture dans toutes les provinces du royaume, à titre de tribunaux d'expertise élargis aux praticiens locaux 55 . Paris, déjà auréolé de son prestige de capitale philosophique, devint le grand centre européen de la pensée économique, avec son intense activité éditoriale, ses revues économiques spécialisées et ses multiples traductions qui firent découvrir en français l'économie politique britannique à l'Europe lettrée (Adam Smith inclus). L'arrivée des physiocrates après la guerre de Sept Ans, dont la production intellectuelle apparaît plus autonome mais aussi plus dogmatique, contribua à perpétuer Paris comme capitale de la science économique... pour un temps seulement.

\section{Ouvrages cités}

\section{SOURCES}

ABEILle, Louis-Paul. 1760. Corps

d'Observations de la Société d'Agriculture, de Commerce et des Arts Établie par les États de Bretagne. Années 1757 \& 1758. Rennes, Jacques Vatar.

- 1763. Lettre d'un Négociant sur la Nature du Commerce des Grains. Marseille, s. n.

AcCarias de SerRIOnNe, Jacques. 1767 [1766]. Les intérêts des nations de l'Europe, développés relativement au commerce. Paris, Dessaint, 2 vol.

Almanach royal pour l'année 1754. 1754. Paris, Le Breton.

Butel-DumONT, Georges-Marie. 1754. Histoire et commerce des colonies angloises dans l'Amérique septentrionale. Londres; Paris, Le Breton-Desaint-Lambert.

CHILD, Josias. 1754 [1668]. Traités sur le commerce et sur les avantages qui résultent de la réduction de lintérêt de l'argent, trad. par V. de Gournay et G. M. Butel-Dumont. Amsterdam; Berlin, Jean Neaulme; Paris, Guérin \& Delatour (éd. orig. Brief Observations concerning Trade and the Interest of Money. Londres, Printed for Elizabeth Calvert...).
CANTILlon, Richard. 1755. Essai sur la nature du commerce en général. Londres, Fletcher Gyles.

Clicquot-Blervache, Simon. 1758. Considérations sur le commerce et en particulier sur les compagnies, sociétés et maîtrises.

Amsterdam, s. n.

Coyer, Gabriel François. 1756. La Noblesse Commerçante, Londres; Paris, Duchesne.

- 1768. Chinki, histoire cochinchinoise qui peut servir à d'autres pays. Londres, s. n.

Defoe, Daniel. 1715. Complete English Tradesman. Londres, printed for J. Rivington, 2 vol.

Dictionnaire de l'Académie française. 1762.

Paris, Vve de Bernard Brunet, 2 vol.

Dupont De Nemours, Pierre-Samuel. 1844 [1808]. «Notice sur les économistes» (Daire et Dussard 1966, 1: 258-261).

FLeURY, Gabriel. 1915. François Véron de Fortbonnais, sa famille, ses actes, ses œuvres (1722-1800). Le Mans, A. de Saint-Denis.

ForbonnAis (VÉRON DE), François. 1754. Élémens du commerce. Leyde, 2e éd., 2 vol. - 1755. Examen des avantages et des désavantages de la prohibition des toiles peintes. Marseille, Carapatria. 
Forbonnais (VÉron DE), François. 1980 [1767]. Principes et observations ceconomiques. Amsterdam, Marc Michel Rey, rééd. Kraus Reprint, Munich.

France. États provinciaux de Bretagne. 1757. Établissement d'une Société d'agriculture, de commerce, et des arts dans la province de Bretagne, par délibération des États. Rennes, chez Joseph Vatar.

Gua de Malves, Jean-Paul. 1757. Discours pour et contre la réduction de lintérest naturel de l'argent. Paris, Grangé.

Herbert, Claude Jacques. 1753. Essai sur la police générale des grains. Londres, s. n.

Journal de commerce. 1759. Janvier. Journal de commerce. 1761. Avril.

Journal ceconomique. 1755. Janvier.

KING, Charles. 1721. The British merchant, or, commerce preserv'd. Londres, J. Darby, 3 vol.

LE Blanc, Jean Bernard. 1754. «Préface du traducteur à Monsieur le docteur Lami, professeur à Florence", in David Hume, Discours politiques de M. Hume. Amsterdam; Paris, Lambert (trad. J.-B. Le Blanc), t. I: v-lviii.

Mably, Gabriel Bonnot de (abbé). 17941795. Du Commerce des Grains, dans Collection Complète des Euvres de l'Abbé de Mably, Paris, Ch. Desbriere, 1794-1795, t. XIII : 242-298.

Mercure de France. 1758. novembre.

Michau de Montaran, Jacques Maris Jérôme. 1754. «Mémoire sur les bleds, par M. D. M. I. D. C.», Journal ceconomique, mars : $122-147$.

Montesquieu, Charles Louis de Secondat (baron de). 1979 [1748]. De l'esprit des lois. Paris, Garnier Flammarion, 2 vol.

MoREllet, André. 1758. Réflexions sur les avantages de la libre fabrication et de l'usage des toiles peintes en France, pour servir de réponse aux divers mémoires des fabriquans de Paris, Lyon, Tours, Rouen, E'c. sur cette matière. Genève; Paris, Damonneville.

- 1759. Réflexions sur differens objets du Commerce, Et en particulier sur la libre Fabrication des Toiles Peintes. Genève, s. n. - 1769. Prospectus d'un nouveau Dictionnaire de commerce. Paris, Estienne frères.
- 1775. Réflexions sur les avantages de la liberté d'écrire et d'imprimer sur les matières de l'administration. Londres; Paris, Frères Estienne.

- 2000 [1821]. Mémoires sur le dix-huitième siècle et sur la Révolution. Paris, Mercure de France (Le Temps Retrouvé).

Notice des principaux articles qui composent la bibliothèque de M. de Dangeul. 1777. Paris, Debure fils aîné.

O'Heguerty, Pierre-André. 1754. Essai sur les intérêts du commerce maritime. La Haye, s. n. - 1757. Remarques sur plusieurs branches de commerce et de navigation. S. 1., s. n.

Plumard de Dangeul, Louis-Joseph (pseud. John Nickolls). 1754. Remarques sur les avantages et les désavantages de la France et de la Grande-Bretagne par rapport au Commerce et aux autres sources de la puissance des États. Leyde, 2e éd.

SAVARY Des Bruslons, Jacques. 1723-1730. Dictionnaire universel de commerce. Paris, Jacques Estienne, 3 vol.

Schelle, Gustave. 1984 [1897]. Vincent de Gournay. Genève; Paris, Slatkine Reprint.

TUCKER, Joshua.1755 [1751]. Questions importantes sur le commerce à l'occasion des oppositions au dernier Bill de naturalisation des protestants étrangers. Londres, E. Gyles, traduction annotée et publiée par Turgot (éd. orig. Reflections on the Expendiency of a Law for the Naturalization of Foreign Protestants. Londres, T. Trye).

Turgot, Anne Robert Jacques. 1997 [1759]. «Éloge de Vincent de Gournay», in A. R. J. Turgot, Formation et Distribution des Richesses. Paris, Garnier Flammarion: 123-153.

UlloA, Bernardo de. 1753 [1740]. Rétablissement des manufactures et du commerce d'Espagne, trad. par L. J. Plumard de Dangeul. Amsterdam; Paris, Vve Estienne et fils (éd. orig. Restablecimiento de las fábricas y comercio español. Madrid, Antonio Marín).

UsTARIZ, Gerónimo de. 1753 [1724?]. Théorie et pratique du commerce et de la marine, trad. par F. Véron de Forbonnais. Paris, Vve Estienne et fils (éd. orig. Theórica $y$ práctica de comercio y de marina. S. 1.). 
VRoIL, Jules de. 1870. Étude sur ClicquotBlervache, économiste du XVIII Siècle. Paris, Guillaumin.

\section{Travaux}

Angolini, Franco et Daniel Roche. 1995. Culture et formations négociantes dans l'Europe moderne. Paris, EHESS (Civilisations et sociétés 91).

Antolne, Michel. 1970. Le Conseil du Roi sous le règne de Louis $X V$. Paris; Genève, Droz (Mémoires et documents publiés par la Société de l'École des chartes).

BAKER, Keith M. 1993 [1990]. Au Tribunal de l'opinion. Essai sur l'imaginaire politique du XVIII siècle. Paris, Payot (Bibliothèque historique) (éd. orig. Inventing the French Revolution. Cambridge-New York, Cambridge University Press).

BALcou, Jean (éd.). 1975. Le dossier Fréron. Correspondances et documents. Genève; Saint-Brieuc, Droz; Presses universitaires de Bretagne.

BECKER, Howard S. 1988 [1982]. Les mondes de l'art. Paris, Flammarion (éd. orig. Art worlds. Berkeley-Los Angeles-London, University of California Press).

Bots, Hans et Françoise WAQUET. 1997. La République des lettres. Paris, Belin (Europe et histoire).

BRIAN, Éric. 1994. La mesure de l'État. Administrateurs et géomètres au XVIII siècle. Paris, Albin Michel (L'évolution de l'humanité).

Charles, Loïc. 1999. «La liberté du commerce des grains et l'économie politique française (1750-1770)», thèse nouveau régime en sciences économiques, université de Paris I.

- 2004. «Le cercle de Gournay: réseaux personnels, institutions et projets politiques », colloque international «Commerce, population et société. Autour de Vincent de Gournay (1748-1758): la genèse d'un vocabulaire des sciences sociales en France», Paris, Ined, 19-21 février.

- 2006. «L'économie politique française et le politique dans la seconde moitié du XVIII" siècle», in Philippe Nemo et Jean Petitot, Histoire du libéralisme en Europe. Paris, Puf (Quadrige): 279-313.

Constant, Jean-Marie. 1991. «Absolutisme et modernité », in Guy Chaussinand-Nogaret (éd.), Histoire des élites en France du XVIе au $X X^{e}$ Siècle. L'honneur, le mérite, l'argent. Paris, Tallandier (Approches): 143-214.

Crouzet, François. 1985. De la supériorité de l'Angleterre sur la France. L'économique et l'imaginaire, $X V I I^{e}-X X^{e}$ siècles. Paris, Perrin (Pour l'histoire).

DAIRE, Eugène et Hippolyte Dussard (éd.). 1966. Euvres de Turgot. Osnabrück, Otto Zeller, 2 vol.

DARNTON, Robert. 1992 [1990]. Gens de lettres, gens du livre. Paris, Odile Jacob (Histoire).

Desrosieres, Alain. 2000 [1993].

La politique des grands nombres. Histoire de la raison statistique. Paris, La Découverte/ Poche (Sciences humaines et sociales).

Dockes, Pierre. 1969. L'Espace dans la pensée économique du XVI au XVIII siècle. Paris, Flammarion (Nouvelle bibliothèque scientifique).

EsPagne, Michel et Michael Werner (éd.). 1988. Transferts. Les relations interculturelles dans l'espace franco-allemand (XVIII et XIXe siècles). Paris, Éd. Recherches sur les civilisations (Travaux et mémoires de la Mission historique française en Allemagne).

Foucault, Michel. 2004. Naissance de la biopolitique. Cours au Collège de France (19781979). Paris, Gallimard/Seuil (Hautes études).

Guarrigues, Frédéric. 1998.

«Les intendants du commerce au XVIII siècle», Revue d'histoire moderne et contemporaine, vol. 45, $\mathrm{n}^{\circ} 3$ : 626-661.

Guery, Alain. 1993. «L'œuvre royale. Du roi magicien au roi technicien", Le Débat, $\mathrm{n}^{\circ} 74$ : 123-142.

HEсHт, Jacqueline. 1964. «Un problème de population active au XVIII siècle en France: la querelle de la noblesse commerçante», Population, vol. 37, $\mathrm{n}^{\circ}$ 2: 267-289.

Hoock, Jochen. 1987. «Discours commercial et économie politique en France au XVIII siècle: l'échec d'une synthèse ", Revue de synthèse, $\mathrm{n}^{\circ} 1: 64-73$. 
IVES, Robin J. 2003. «Political publicity and political economy in eighteenth century France ", French History, vol. 17, n ${ }^{\circ}$ 1: 1-18.

JEANNIN, Pierre. 1989. «Les manuels de pratique commerciale imprimés pour les marchands français (XVIe-XVIII siècle)», in François M. Crouzet (éd.), Le Négoce international $X \mathrm{II}^{e}-X X^{e}$ siècle. Paris, Economica (Économies et sociétés contemporaines): 35-59. - 1995. «Distinction des compétences et niveaux de qualification: les savoirs négociants dans l'Europe moderne", in Angolini et Roche 1995 : 363-397.

KaPLAN, Steven L. 1986 [1976]. Le pain, le peuple et le roi. Paris, Perrin (éd. orig. Bread, Politics and Political Economy in the Reign of Louis $X V$. The Hague, M. Nijhoff).

LARRERE, Catherine.1992. L'invention de l'économie au XVIII' siècle. Du droit naturel à la physiocratie. Paris, Puf (Léviathan).

LebEaU, Christine. 2004. «Éloge de l'homme imaginaire. Recherches sur la construction de la figure de l'administrateur au XVIIIe siècle», Le Genre humain, ${ }^{\circ}$ 42: 99-116.

Lemercier, Claire. 2005. «Analyses de réseaux et histoire», Revue d'bistoire moderne et contemporaine, vol. $52, \mathrm{n}^{\circ} 2$ : 88-112.

LeSPAGNOL, André. 1995. «Modèles éducatifs et stratégies familiales dans le milieu négociant malouin aux XVIIe et XVIIIe siècles: les ambiguités d'une mutation", in Franco Angolini et Daniel Roche (éd.), Culture et formations négociantes dans l'Europe moderne. Paris, EHESS (Civilisations et sociétés): 257-274.

Mercklé, Pierre. 2004. Sociologie des réseaux sociaux. Paris, La Découverte (Repères).

MeysonNiER, Simone. 1989. La balance et l'horloge. La genèse de la pensée libérale en France au XVIII siècle. Montreuil, Éd. de la Passion.

MinarD, Philippe. 1998. La fortune du colbertisme. Etat et industrie dans la France des Lumières. Paris, Fayard.

Mousnier, Roland. 1979. «La fonction publique en France, du début du XVIe siècle à la fin du XVIIe siècle», Revue historique, vol. 261: 321-335.

MurPHY, Antoin. 1986. «Le développement des idées économiques en France (1750-
1756)", Revue d'histoire moderne

et contemporaine, $\mathrm{n}^{\circ}$ 33: 521-541.

Perrot, Jean-Claude. 1992. Une histoire

intellectuelle de léconomie politique, ХVII--ХИIIए

siècles. Paris, EHESS (Civilisations et sociétés).

REY, Alain (éd.). 1992. Dictionnaire historique de la langue française. Paris, Le Robert.

Roche, Daniel. 1988. Les républicains des lettres. Gens de culture et Lumières au XVIII siècle. Paris, Fayard (Nouvelles études historiques). - 1993. La France des Lumières. Paris, Fayard.

SCHUMPETER, Joseph A. 1983 [1954].

Histoire de l'analyse économique, vol. 1: L'Âge des fondateurs. Paris, Gallimard (Bibliothèque des Sciences Humaines) (éd. orig. History of economic analysis, Oxford, Oxford UP).

SMITH, David K. 2003. «Structuring Politics in Early Eighteenth Century-France: the Political Innovations of the French Council of Commerce", The Journal of Modern History, vol. 74, n $^{\circ} 3$ : 490-537.

SMITH, Jay M. 2005. Nobility Reimagined. The Patriotic Nation In Eighteenth-century France. Ithaca and London, Cornell University Press.

STEINER, Philippe. 1988. Sociologie de la connaissance économiques. Essai sur les rationalisations de la connaissance économique (1750-1850). Paris, Puf (Sociologies).

TARRADE, Jean. 1996. «Liberté du commerce, individualisme et État. Les conceptions des négociants français au XVIII" siècle», Cahiers d'économie politique, n' 27-28: 175-190.

THÉRÉ, Christine. 1984. «Éléments d'une sociologie des auteurs économiques de langue française (1715-1776)», Economia, n ${ }^{\circ} 1$ : 11-33.

TsudA, Takimi (éd.). 1983. Traités sur le commerce de Josuah Child avec les remarques inédites de Vincent de Gournay. Tokyo, Economic Research n ${ }^{\circ} 20$, Kinokuniya Company. - 1993. Mémoires et lettres de Vincent de Gournay. Tokyo, Kinokuniya Company LTD.

VAN DAMME, Stéphane. 1998. «La sociabilité intellectuelle. Les usages historiographiques d'une notion». Hypothèses 1997, Paris, Publications de la Sorbonne: 123-132. - 2005. Paris, capitale philosophique. De la Fronde à la Révolution. Paris, Odile Jacob (Histoire). 


\section{Notes}

1. Sur les officiers, voir Constant (1991), et sur la fonction publique d'Ancien Régime, voir Mousnier (1979).

2. Accarias de Serrionne (1767: 26), animateur d'une revue économique qui a tôt promu la "Science du Commerce», fait allusion ailleurs à ce "petit nombre de François, également philosophes $\&$ citoyens, [qui] commencèrent il $\mathrm{y}$ a quelques années, à imiter les écrivains anglois. Ils traduisirent d'abord leurs modèles, \& les ont bientôt surpassés en beaucoup de choses».

3. Ainsi Morellet (2000: 72-73) ne retient que Malesherbes, Turgot, Dangeul, Forbonnais, Trudaine et lui-même. Dupont (1844: 260) ajoute à cette liste «Herbert, d'Ynvau, Boisgelin, Cicé, Dangeul», ainsi que quelques étrangers (ibid.: 315).

4. Loïc Charles (2004), qui dénombre cependant cinq «réseaux» du collectif (parlementaire, provincial, négociant, littéraire et administratif).

5. Sur les divers usages de la notion de «réseau » en histoire et en sociologie, voir Lemercier 2005 et Mercklé 2004.

6. Le "personnel de renfort» désigne chez Howard S. Becker (1988) tous les médiateurs qui travaillent à la production d'une œuvre, fût-elle signée par un seul auteur, des collaborateurs jusqu'au public en passant par les secrétaires, éditeurs, imprimeurs... tous engagés dans des rapports de coopération (non exempts de rapports de pouvoir). Nous empruntons à Frédérique Matonti la transposition de cette approche du «monde des arts» au «monde des idées», mise en œuvre dans son séminaire d'histoire sociale des idées politiques; qu'elle ne soit bien sûr nullement tenue pour responsable de nos errements.

7. En se limitant à la décennie 1750 et en comptant la traduction de Joshua Tucker, les articles «Foire» et «Fondation» pour l'Encyclopédie, ainsi que l'«Éloge de Gournay».

8. Nous avons consulté pour l'essentiel deux revues «savantes» (le Journal de Trévoux et le Journal des savants), deux revues mondaines (le Mercure de France et le Journal de Verdun), et deux revues «économiques» spécialisées (le Journal de commerce et le Journal œconomique).

9. Sur la vie de Gournay, la source première reste Turgot (1997). Pour les biographies, voir Gustave Schelle (1984) et celles, plus récentes et bien documentées de Takimi Tsuda (1983: 445-485) et de Simone Meyssonnier (1989 : 168-188).
10. Pour un bon aperçu du "projet» politico-économique de Gournay, voir son rapport «d'espionnage» adressé à Maurepas vers 1747-748 (Tsuda 1993 : 3-38).

11. Gournay possédait cent soixante-neuf livres d'économie et de philosophie politique, alors que la bibliothèque standard des intendants du commerce comportait fort peu d'ouvrages économiques ou de codes de commerce (Guarrigues 1998: 634-636).

12. Entre autres: Claude Carlier, Jean Hellot, Jean Rodolphe Perronet, Nicolas Desmarets, Jacques Vaucanson, John Holker. Ces deux derniers collaborèrent à plusieurs reprises avec Gournay au Bureau du commerce; et l'on trouve dans les papiers de Gournay conservés à la bibliothèque municipale (par la suite BM) de Saint-Brieuc (Ms 82) deux mémoires de Holker sur les manufactures et les toiles peintes.

13. Sur sa vie, voir Vroil 1870: I- LIX.

14. Clicquot de Blervache (1758).

15. Voir la biographie de Gabriel Fleury (1915).

16. On trouve dans les papiers de Gournay le manuscrit de «Deux Projets de Refonte du billon» (BM de Saint-Brieuc. Ms. 82, ff. 70-75), suivi d'une brève «réponse» - semble-t-il écrite de la main de Gournay - au «Préambule du projet de M. de La forbonaye» (ibid. ff. 79-81).

17. Voir son signalement par la police en 1752 dans Darnton 1992 : 82.

18. Almanach royal pour l'année 1754 (1754: 195). À toutes fins utiles, le publiciste Élie Fréron signale à l'inspecteur Joseph Hémery «que le Rétablissement des Manufactures et du Commerce d'Espagne, imprimé chez les frères Estienne, ouvrage traduit de l'espagnol, est de M. Dangeul qui demeure rue Saint-André des Arts chez M. Bellanger Avocat Général à la Cour des Aides? Dangeul est le fils d'un riche négociant du Mans [...]», dans Balcou (1975 : 100, lettre du 17 septembre 1753).

19. Forbonnais précise au diplomate Hennin à qui il avait recommandé de rencontrer Dangeul en Italie, que celui-ci «a voïagé dans ce paîs la dans d'autres vuës que celles du plaisir des arts» (Bibliothèque de l'Institut - BI par la suite. Ms. 1259, ff. 286-287. Lettre du 11 septembre 1758).

20. Bibliothèque de l'Arsenal. 6496, ff. 112-122. Et pour l'inventaire de la bibliothèque de Dangeul voir Notice des principaux articles... (1777). 
21. Voir son dossier aux Archives nationales (par la suite $\mathrm{AN})$. F17/1288.

22. Ce livre «a été traduit en 1754 par MM. Gournay et Butel-Dumont", lit-on au crayon à la première page du manuscrit (BM de Saint-Brieuc. Ms 81, f. 1)

23. BI. Ms 1266, ff. 446-447. Lettre du 23 août 1756 (citée par L. Charles 1999 : 129).

24. Par exemple: Journal de commerce (janvier 1759: 39).

25. Sur ce corps de fonctionnaires d'Ancien Régime, et le rôle d'Abeille et Clicquot-Blervache dans la difficile promotion du libéralisme économique au sommet de l'État, voir Minard 1998. À côté des employés de bureaux, l'inspection recruta un nouveau type d'agents publics, des experts spécialisés (ibid.: 31 ).

26. Sur cette «stratégie éditoriale», nous suivons L. Charles (1999 : partie II).

27. Pour un bilan historique éclairé sur la «République des Lettres», voir Bots et Waquet 1997.

28. Dans une lettre à Malesherbes (14 août 1756), l'abbé Nicolas Charles Joseph Trublet désapprouve Coyer, "malgré ma déférence pour mon ami et compatriote [de Saint-Malo] Mr de Gournay " (Balcou 1975 : 179, lettre du 17 septembre 1753), suggérant que celui-ci est derrière l'affaire. Pour l'histoire de cette querelle, voir Hecht 1964 et Smith 2005 : chap. III.

29. Voir la lettre de Gournay à Morellet (Tsuda 1993 : 104).

30. Turgot 1997 : 143; France. États provinciaux de Bretagne. 1757: 4.

31. Remarque congruente de Forbonnais (1754, 1: 85). On trouve l'expression «science du commerce» sous la plume du marquis d'Argenson (qui approuvait l'action de Gournay), mais toujours pour affirmer l'impossibilité de la dite science, assimilée à la vieille «police».

32. David K. Smith donnera à lire la correspondance diplomatique de Gournay dans l'édition prochaine des actes du colloque international de l'Ined consacré à l'intendant en février 2004 («Commerce, population et société, autour de Vincent de Gournay. La genèse d'un vocabulaire des sciences sociales en France»), ce qui devrait jeter un nouvel éclairage sur ces années de «politisation» du négociant.

33. BM de Saint-Brieuc. Ms. 84, fol. 24, 71, 175.

34. Ibid. Ms. 84, f. 323.

35. Ibid. Ms. 82.
36. Ibid. Ms. 83, "Mémoire sur les changes» (1752), f. 341.

37. «La maxime fondamentale de tout commerce que l'on veut rendre florissant: liberté et protection» (Tsuda $1983:$ 421).

38. BM de Saint-Brieuc. Ms 82, f. 322 . Voir aussi Tsuda (1983 : 184-187). L'Acte de navigation fut traduit par O'Heguerty (1754: 217-240) puis Butel-Dumont en 1760.

39. «[...] lorsque j’ai désiré la charge d'Intendant du commerce, j’y ai été poussé par l'espoir de rapprocher un peu plus le commerce et les négociants des personnes en place», écrit-il à Trudaine dans sa lettre de démission (Tsuda 1993 : 106).

40. Antoine (1970 : 222). Frédéric Guarrigues (1998) a consacré une étude fouillée à ce corps.

41. BI. Ms. 1259, ff. 279-281. Lettre du 19 juin 1758.

42. Ibid. ff. 282-283. Lettre du 30 juin 1758.

43. Remarque similaire de Plumard de Dangeul (1754: 4-5).

44. Morellet (1769: 357; $1775: 10)$.

45. Gournay communiqua le manuscrit à un philosophe aussi peu favorable au laisser-faire que Mably (1794-1795 : 294). Forbonnais l'annota, ainsi que Butel-Dumont qui écrit à Malesherbes: «J'ai mis aussi dans ce paquet les observations de M. de Gournay sur le mémoire concernant les laines [chap. vIII des Traités] que vous m'avez chargé de parapher». Bibliothèque nationale de France (par la suite BNF). Fr. 22147, ff. 38-39. Lettre du 28 janvier 1755. Morellet (1769: 357) le récupéra à la mort de Gournay.

46. Sur les dictionnaires de commerce, voir Perrot (1992: 97-125).

47. Analyse convergente de Turgot (1997: 125), voir Hoock (1987) sur ce point.

48. BNF. NAF 3344, ff. 117. Lettre de Jean Larue, 26 février 1746 (nous soulignons).

49. Sur cette controverse, voir Meyssonnier (1989 : 267-72).

50. Sur l'opinion publique comme sociabilité politique d'une nation raisonnable, voir Baker (1993: chap. VI). Sur le rôle de l'économie politique dans la genèse de l'opinion publique des Lumières, voir les études de Robin Ives (2003) et Loïc Charles (2002: 24-25; 2004).

51. Comme toute le ministère français au cours du XVIII siècle, paniqué et admiratif devant les réussites anglaises, qu'il s'exagérait cependant beaucoup (Crouzet 1985 : chap. V). 
52. Lettre à Trudaine du 19 décembre 1752 (Tsuda $1993: 155$ ).

53. Les travaux exemplaires d'Alain Desrosières et d'Éric Brian sur la genèse de la statistique et de la démographie ont montré toute la fluidité des frontières entre savants, experts, administrateurs et État sous l'Ancien Régime.
54. Sur la formation d'une «cité savante» d'économistes en France au XVIII e siècle, de Boisguilbert à Quesnay, voir les analyses de Jean-Claude Perrot (1992: 77-86).

55. AN. F/12/149, f. 194. Circulaire aux intendants (22 août 1760). 